\title{
The Analysis of Mechanical Vibrations and Acoustic Pressure Level of a Transformer Model
}

\author{
T. Boczar*, S. Borucki, A. Cichon And M. LorenC \\ Faculty of Electrical Engineering and Automatics, Technical University of Opole \\ Mikołajczyka 5, 45-271 Opole, Poland

\begin{abstract}
The paper presents the results of vibroacoustic investigations and the measurements of the acoustic pressure level (noise) of a transformer model. The measurements were taken at a full transformer load in two operation conditions: at a producer packeted core and at unpacketed core. Unpacketing consisted in loosing the screws pressing the particular core plates. Mechanical vibrations were registered and the acoustic pressure level was measured. The vibroacoustic analysis results are presented in the form of frequency spectra and the results of the acoustic analysis of the transformer under study are shown by determining corrected values of the acoustic pressure level and by a frequency analysis.
\end{abstract}

PACS numbers: 43.58.Wc, 43.58.Fm

\section{Introduction}

Modern approach of electric power enterprises to the management of the network assets accumulated, especially power transformers, is based on a multi-parameter analysis of the units under study [1-4]. An overall assessment of a transformer may be carried out based on several parameters, including its current significance in an electric power system and its impact on environment. Transformer operation is more and more often connected with the problem of an excessive emission of noise into environment. This phenomenon becomes even more valid if a transformer station is located in the vicinity of living quarters. The problems with exceeding allowable values of acoustic power around a transformer usually refer to the units of older constructions. The sources of sound are not only magnetostriction phenomena taking place in the core or cooler spinning fans but also windings which vibrate due to electrodynamic forces and operation of the pumps forcing oil flow.

${ }^{*}$ corresponding author; e-mail: t.boczar@po.opole.pl 
The paper presents the results of vibroacoustic investigations and measurements of the acoustic pressure level around a dry transformer model, in which core damage, consisting in its partial unpacketing, was modelled.

\section{Characteristics of the measuring setup}

The measurements were taken on the transformer model, the view of which is shown in Fig. 1. The transformer under study was characteristic of the following nominal data:

- type: ET3S-20,

- power: $20 \mathrm{kVA}$,

- rated voltage GN: $3 \times 400 \mathrm{~V}$,

— rated voltage DN: $3 \times 20 \mathrm{~V}$,

- rated current GN: $17.1 \mathrm{~A}$,

— rated current DN: $333 \mathrm{~A}$,

— type of work: S1.

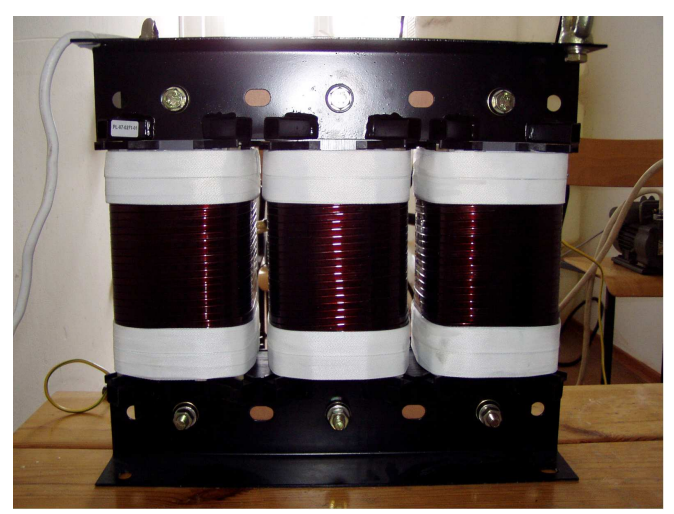

Fig. 1. Low-voltage transformer model under study.

Mechanical vibrations of the transformer model were measured with an accelerometer type 752-10 by Endevko company, which was attached to the core with a magnet and the view of which is shown in Fig. 2a. Sensitivity of the measuring transducer was $1.021 \mathrm{mV} /\left(\mathrm{m} \mathrm{s}^{2}\right.$ ) (for $100 \mathrm{~Hz}$ ). The signal received by the transducer was passed onto the input of a low-noise measuring amplifier from Nexus 2693 family by the firm Brüel \&Kjær, shown in Fig. 2b. In order to get separated from disturbances transferred by the low-voltage net $230 \mathrm{~V}$, the amplifying system was supplied from the internal battery source during the measurements. A computer equipped with a measuring card type $\mathrm{CH} 3160$ by the firm Acquitek and specialized AcquiFlex software were used for observation and registration of the vibration signals.

The measurements of the acoustic pressure level were taken with a meter type $945 \mathrm{~A}$ by the firm SVAN. This appliance is used for taking measurements of 

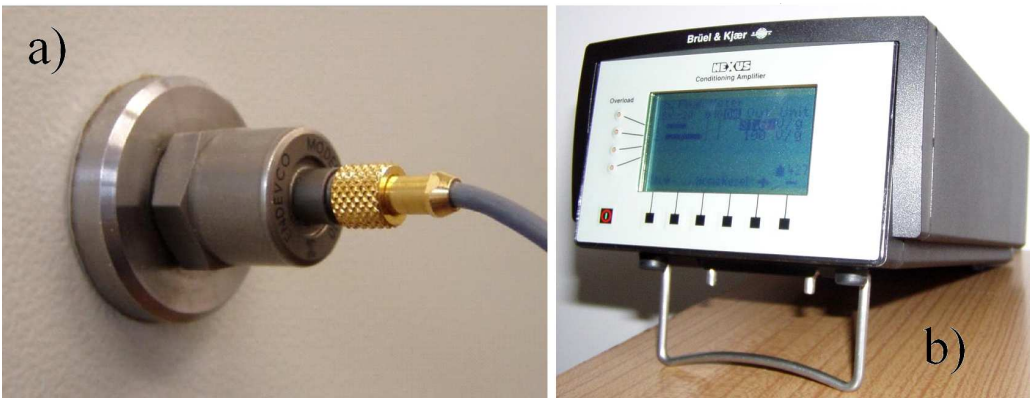

Fig. 2. View of the transducer (a) and the measuring amplifier (b).

the sound level with accuracy corresponding to class 1 and the frequency analysis of the acoustic signals in the band $1 \mathrm{~Hz}-20 \mathrm{kHz}$. The measuring tool makes it also possible to perform: the tone analysis, volume measurement, octave and tierce analysis, fast Fourier transform (FFT) analysis and detecting discrete tones of the acoustic signal measured. Figure 3 shows the appearance of the meter used.

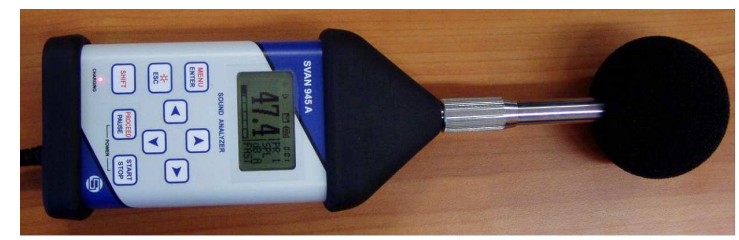

Fig. 3. View of the meter of the acoustic pressure level.

\section{Methodology of measurement taking}

During the measurements, the transformer under study was loaded with rated current using a water resistance making adjustment of the current values in the range from 0 to $340 \mathrm{~A}$ possible. The investigation was carried out in the stages: the first stage contained analyses of the transformer vibrations and the acoustic pressure level at a packeted core, and the second stage referred to measurements at an unpacketed core. Mechanical vibrations were registered with a transducer placed on the core, and the acoustic pressure level was measured in the distance of $1 \mathrm{~m}$ away from the transformer under study.

\section{Analysis of the results obtained}

In the result of the measurements taken, the values of the corrected acoustic pressure levels for two operation conditions of the transformer under study were determined, which are shown in Table I.

Operation of a transformer with a properly pressed core is characteristic of the value of the acoustic pressure level lower by $12.2 \mathrm{~dB}$ than in the case when 
TABLE

Mean level of the acoustic pressure registered around transformers with packeted and unpacketed cores.

\begin{tabular}{c|c}
\hline \hline \multicolumn{2}{c}{ Mean level of acoustic pressure in $\mathrm{dB}$} \\
\hline packeted core & unpacketed core \\
\hline 51.6 & 63.8
\end{tabular}

the core has become loose. This difference in the values obtained indicates a significant influence of the construction and proper packeting of the transformer core on the noise emitted into surrounding. In order to examine the influence of the modelled core damage on the participation of the particular frequency components, the measurement of the acoustic pressure level was supplemented by an analysis in the frequency domain, which was carried out by using a FFT and an octave analysis $[5,6]$. The results of the frequency analysis are shown in Figs. 4 and 5.

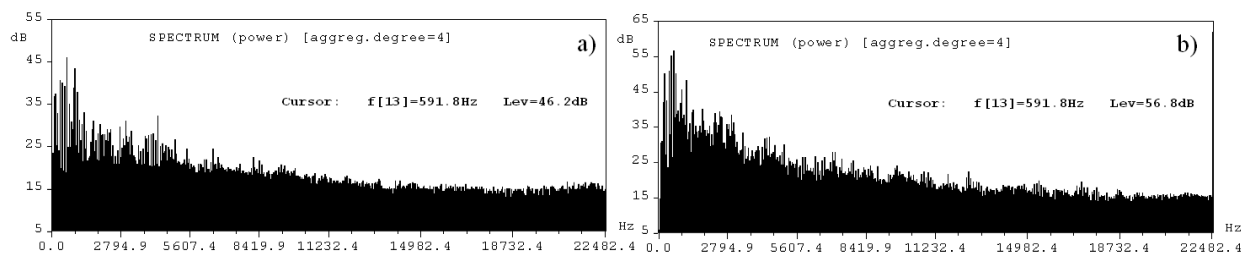

Fig. 4. The results of the FFT analysis of the acoustic pressure level around the transformer: (a) with the packeted core, (b) with the unpacketed core.
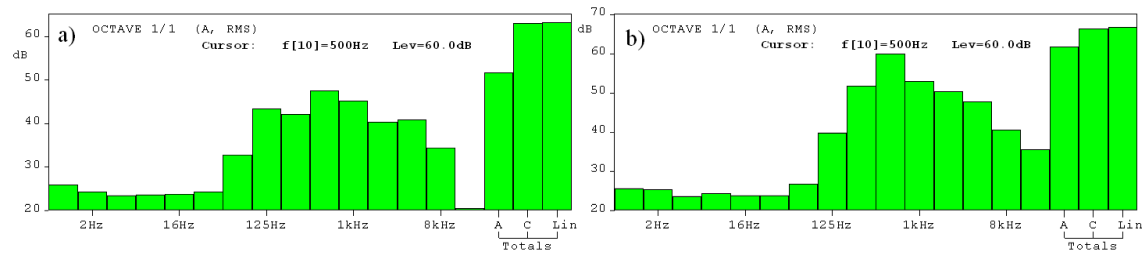

Fig. 5. The results of the octave analysis of the acoustic pressure level around the transformer: (a) with the packeted core, (b) with the unpacketed core.

Analyzing the runs of the frequency spectra as well as the column diagrams showing the results of the octave analysis, it can be observed that participations of the particular frequencies are relatively constant and do not depend on the modelled core damage. Significant differences can be observed analyzing amplitude values of the particular frequency components. Bigger amplitudes correspond to the results determined for the transformer with the loose core. In order to determine frequency ranges, based on which it is possible to discover core damage in a transformer, it is necessary to carry out vibroacoustic investigations. The 
measurement results of the mechanic vibrations of the transformer under study are shown in Fig. 6.

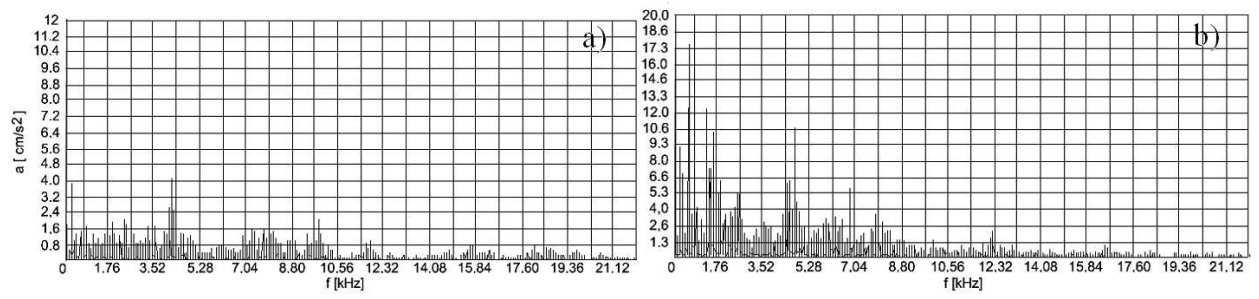

Fig. 6. Frequency spectra of transformer vibrations: (a) packeted core, (b) unpacketed core.

Amplitude values, determined based on the measurement of the vibration acceleration, differ both in the range of dominant frequencies and in amplitude. In the case of the loose core, a significant participation of components for frequencies from the range from 0 to about $5 \mathrm{kHz}$ can be observed.

\section{Summing-up}

An excessive value of the acoustic pressure level occurring around transformers is a significant problem for both professional power engineering and services involved in environment protection $[7,8]$. Over the years the level of the acoustic pressure emitted by power transformers into environment is not constant but is subject to changes. This can be caused either by a significant wear of cooling appliances or changes taking place in a transformer core. The works on noise reduction presently concentrate on seeking new technical solutions, the aim of which is limiting the value of the acoustic pressure level emitted by such appliances as pumps or coolers. Increasing the noise level above the values provided at the stage of a transformer construction can be the cause of non-compliance with environmental standards. Therefore it is vital that the acoustic pressure level be monitored during periodical check-ups, especially around high-power transformers.

The research results presented in this paper indicate a significant influence of the core construction on the value of the acoustic pressure level occurring around a transformer. These results prove that the assessment of the transformer core condition should be an indispensable element of a transformer diagnostics as potential failures can influence not only technical parameters but also increase the noise level emitted into surrounding.

\section{Acknowledgments}

The research work was financed with the means assigned for science as research-development project No. R01 00601 and individual research project No. N511 019 31/3638. 


\section{References}

[1] A. Carlson, J. Fuhr, G. Schemel, F. Wegscheider, ABB - Testing of Power Transformers, Pro Print, Düsseldorf 2003.

[2] T. Boczar, S. Borucki, A. Cichoń, M. Lorenc: in Proc. 37th Int. Conf. and Exhibition, Defektoskopie 200\%, Praha 200\%, Eds. M. Kořenska, P. Mazal, Brno University of Technology, Brno 2008, p. 45.

[3] T. Boczar, M. Lorenc, D. Zmarzły, in: Proc. 14th Int. Congress on Sound Vibration, Cairns (Australia) 2007, 1-8, Abstract 316, p. 1.

[4] T. Boczar, S. Borucki, A. Cichoń, Arch. Acoust. 32 (Supplement), 291 (2007).

[5] T. Boczar, S. Borucki, A. Cichoń, Mol. Quant. Acoust. 28, 13 (2007).

[6] IEEE Standards, Test Core for Liquid-Immersed Distribution, Power and Regulating Transformers, IEEE Std. C57.12.90 (1999).

[7] Order of Environment Ministry since 29.07.2004 (Dz. U. since 13.08.2004) (in Polish).

[8] T. Boczar, S. Borucki, A. Cichoń, M. Lorenc, Mol. Quant. Acoust. 28, 45 (2007). 